\title{
Spontaneous recurrent hematuria and hematospermia: Unique manifestations of von Willebrand disease type $I$. Case report
}

\author{
Daniele Minardi ${ }^{1}$, Anna Rita Scortechini ${ }^{2}$, Giulio Milanese ${ }^{1}$, Pietro Leoni ${ }^{2}$, Giovanni Muzzonigro ${ }^{1}$ \\ ${ }^{1}$ Department of Clinic and Specialistic Sciences, Urology; ${ }^{2}$ Department of Clinic and Molecular Sciences, Institue of Hematology, \\ Polytechnic University of the Marche Region, A.O. Ospedali Riuniti, Ancona, Italy.
}

\begin{abstract}
Summary In this report we describe the case of a patient with unrecognized von Willebrand disease (vWD), in whom the only presenting symptoms were spontaneous and recurrent hematuria with bladder tamponade, associated with recurrent hematospermia. The diagnosis was made only after several admissions to the hospital. We suggest to include coagulopathies such as vWD as part of the evaluation in patients with unexplained genito-urinary bleeding.
\end{abstract}

KEY WORDS: von Willebrand disease; Hematuria; Hematospermia.

Submitted 8 March 2015; Accepted 21 May 2015

\section{INTRODUCTION}

von Willebrand disease is the most common congenital bleeding disorder in the USA; population-based prevalence studies suggest that the disorder affects more than $1 \%$ of individuals screened, with little difference among racial and ethnic groups (1). A prolonged bleeding time with a normal platelet count is the most important laboratory abnormality. While in 1926 von Willebrand described a novel bleeding disorder, only in the 1950s it was demonstrated that the prolonged bleeding time is associated with reduced Factor VIII, and in the 1970s it was clarified that the deficiency of a new factor, called von Willebrand Factor and different from Factor VIII, was actually responsible for the disease. The disorders arises from qualitative (type 2 vWD) or quantitative (type 1 and 3 vWD) aberrations in the production or clearance of von Willebrand factor (vWF)(3). This large multimeric glycoprotein is synthetized by endothelial cells and megakariocytes, and serves as a promoter of platelet aggregation and a carrier of coagulation factor VIII:C. It is an extremely heterogeneous disorders with up to 20 distinct subtypes reported (2). vWF is essential for platelet-subendothelium adhesion and platelet-toplatelet interactions as well as platelet aggregation in vessels; vWF is the specific carrier of Factor VIII in plasma, in this way protecting it from proteolytic degradation, prolonging its half-life in circulation and efficiently localizing it at the site of vascular injury. Type I vWD is the most common form, accounting for up to $80 \%$ of reported cases, and is generally transmitted as an autosomal dominant disorder. Mucocutaneous bleeding is the most common symptom in these patients, and typically presents as epistaxis, easy bruising, menorrhagia, gingival bleeding and post-traumatic or postsurgical bleeding (4)

To date very few cases of spontaneous hematuria and hematospermia have been reported $(5,6)$; they were secondary to trauma such as self-instrumentation, catheterization, falls or straddle injuries.

In this report we describe a patient with unrecognized vWD, in whom the only presenting symptoms were spontaneous and persistent hematuria and hematospermia.

\section{Case report}

A 34 years old male patient, with chronic adrenal failure under substitutive therapy, came to our observation because of spontaneous and persistent hematuria and hematospermia, recurring since a few years; the hematuria was so important that in few occasions he underwent to acute urinary retention and bladder tamponade. He was already been evaluated in other Urologic Units, where investigations were performed; he had renal, bladder, and prostatic ultrasound, abdominal CT scan and cystoscopy, that showed normal morphology and function of the urinary tract; urine and semen colture did not show any infection.

In the family history, no hemorrhagic disease was present. He was admitted from Emergency to our Department of Urology for further investigations and treatment, after another episode of hematuria with bladder tamponade and acute urinary retention.

We performed routine blood exams, that were normal, and a urethrocystoscopy, that did not show any abnormality; renal and bladder ultrasound was normal, while prostatic ultrasound showed only a slight periurethral congestion and oedema, with symmetric seminal vesicles. An hematologic consultation was then requested; coagulation parameters PT, PTT, Fibrinogen and Platelet count were normal, as well as the platelet agglutination test with Ristocetin; in the suspicion of an hematologic disorder further investigations were performed; it was pos- 
sible to detect a deficit of von Willebrand factor (33\%, with normal values being in between 70 and $150 \mathrm{U} / \mathrm{dl}$ ) and of the Ristocetinic factor (31\% with normal values being in between 58 and $166 \mathrm{U} / \mathrm{dl}$ ), while the Factor VIII resulted normal $(86 \%$ with normal values being in between 70 and $200 \mathrm{U} / \mathrm{dl}$ ).

The diagnosis was confirmed by the Desmopressin test; the positive response to it gave evidence of type I von Willebrand disease.

\section{Discussion}

Von Willebrand disease is a diagnostic entity recognized for at least 80 years, and considered the most common inherited disorder. Mutations at the vWF locus on chromosome 12 leads to $\operatorname{vwD}(2,7)$. Although generally considered to be inherited as an autosomal dominant trait, vWD inheritance may be more complex, including compound heterozygosity, which may contribute to phenotypic variability seen in the disease (1). Further, extragenic factors including $\mathrm{ABO}$ blood groups (8), adrenergic states (9), hormones (10) and inflammatory states (11) may cause fluctuation in vWF levels and mask a vWD diagnosis. Spontaneous genitourinary bleeding is very rare in the general population, and we did not find in the literature a description of cases of bladder tamponade as a presenting symptom of vWD. When spontaneous bleeding occurs, it is mild and in most cases the symptoms resolve spontaneously without the need of invasive treatment. We decided to publish this unusual case report of a patient presenting with recurrent gross hematuria and bladder tamponade; we want to draw attention to the presence of hematologic disorders in cases of unexplained recurrent genito-urinary bleeding; we stress the importance of including the evaluation for existing coagulopathies, such as vWD, in such cases.

\section{References}

1. Ziv O, Ragni MV. Bleeding manifestations in males with von Willebrand disease. Haemophilia. 2004; 10:162.

2. Nichols WC, Ginsburg D. Willebrand disease. Medicine 1993; 76:893.

3. Rodeghiero F, Castaman G. Congenital von Willebrand disease type I: definition, phenotypes, clinical and laboratory assessment. Best Pract Res Clin Haematol. 2001; 14:321.

4. Armenian S, Raffel JL, Nugent DJ, Young G. Painless urethral bleeding: an unusual presentation of von Willebrand disease. Haemophilia. 2003; 9:332.

5. Benton O, Lazarchick J, Orak JK, Turner WR. Use of a bleeding time determination in the evaluation of unexplained hematuria. J Urol. 1987; 137:527.

6. Lemesh RA. Case report: recurrent hematuria and hematospermia due to prostatic teleangiectasia in classic von Willebrand disease. Am J Med Sci. 1993; 306:35.

7. Ginsburg D, Handin RI, Bonthron DT, et al. Human von Willebrand factor ( $\mathrm{vWF}$ ): isolation of complementary DNA (cDNA) clones and chromosomal localization. Science 1985; 228: 1401.

8. Gill JC, Endres-Brooks J, Bauer PJ, et al. The effect of ABO blood group on the diagnosis of von Willebrand disease. Blood. 1987; 69:1691.

9. Rickles FR, Hoyer LW, Rick ME, Ahr DJ. The effects of epinephrine infusion in patients with von Willebrand disease. J Clin Invest. 1976; 57:1618.

10. Liu L, Wang X, Lin Z, Wu H. Elevated plasma levels of vWF:Ag in hyperthyroidism ar mediated through adrenergic receptors. Endocr Res. 1993; 19:123.

11. Bloom AL. The biosynthesis of factor VIII. Clin Haematol. 1979; 8:53.

\author{
Correspondence \\ Daniele Minardi, MD \\ d.minardi@gostec.net \\ Giulio Milanese, MD \\ Giovanni Muzzonigro, MD \\ Department of Clinic and Specialistic Sciences, Urologic Clinic \\ Polytechnic University of the Marche Region, A.O. Ospedali Riuniti \\ Via Conca 71 - 60020 Ancona, Italy \\ Anna Rita Scortechini, MD \\ Pietro Leoni, MD \\ Department of Clinic and Molecular Sciences, Institute of Hematology \\ Polytechnic University of the Marche Region, A.O. Ospedali Riuniti \\ Via Conca 71 - 60020 Ancona, Italy
}

